\title{
On the Finite Model Property for Kripke Models
}

By

\author{
Hiroakira ONO
}

This work is a sequel to [4]. The familiarity with the results and terminology of $[4]$ is presupposed.

It is a well-known fact that the intuitionistic propositional logic (abbreviated as $L J$ ) has not a finite characteristic model. But, Jaśkowski proved that there is a monotonic descending sequence of finite models which converges to $L J$. Using the notation in [4], we can restate this result as follows; there are finite (pseudo-Boolean) models $P_{i}(i \in I)$ such that $L J)\left(\bigcap_{i \in I} P_{i}\right.$.

Now, let's consider the following problem. Let $L$ be any intermediate logic. Are there finite pseudo-Boolean models $P_{i}(i \in I)$ such that $L \backslash \bigcap_{i \in I}$ $P_{i}$ ? If this problem is solved affirmatively for a $\operatorname{logic} L$, we say $L$ has the finite model property, following Harrop's terminology [1]. In [1], it is proved that if a logic $L$ is finitely axiomatizable and has the finite model property then $L$ is decidable. It is an interesting problem whether all intermediate logics are decidable. But we don't know even whether all intermediate logics have the finite model property. In this paper, we show that this problem can be reduced to the following problem presented in [4]: Has any intermediate logic a characteristic Kripke model?

We extend the result to that for logics between Johansson's minimal logic and the classical propositional logic, and show that the logics whose decidability are not known in [5] are decidable. Now, we state our main theorem.

Received December 14, 1970. 
Theorem 1. If $M$ is a Kripke model then $L(M)$ has the finite model property.

Proof. Suppose that $A$ is a formula such that $A \notin L(M)$. We construct a finite Kripke model $N$ such that 1) $A \notin L(N)$ and 2) $L(M) C$ $L(N)$. We remark that the finite Kripke model constructed by the method of Lemmon [3] or Segerberg [5] satisfies 1) but not 2) in general. But the finite Kripke model constructed by our method always satisfies both 1) and 2).

By the assumption, there is an $M$-valuation $W$ such that $W(A, a)=f$ for some $a \in M$. Let $K$ be the set of all subformulas of $A$. For such $W$ and $K$, define a binary relation $\equiv$ on $M$ by

$x \equiv y$ if and only if $W(B, x)=W(B, y)$ for any $B \in K$.

It is easy to verify that $\equiv$ is an equivalence relation on $M$ and that the quotient set $M / \equiv$ of $M$ (with respect to $\equiv$ ) is finite. Now we define essential chains as follows.

Definition 2. A sequence $\alpha\left(=<a_{1}, \ldots, a_{n}>\right)$ of elements of $M$ is said to be a chain (of $M$ ), if $a_{i}<a_{i+1}$ for any $i$ such that $1 \leq i<n$. In such a case, we say $\alpha$ is of length $n$ and write $\operatorname{lh}(\alpha)=n$. We write $(\alpha)_{i}$ for $a_{i}$. That is, $\alpha=\left\langle(\alpha)_{1}, \ldots,(\alpha)_{l h(\alpha)}\right\rangle$. A chain $\alpha$ is said to be essential (with respect to $\equiv$ ) if $\alpha$ satisfies the following three conditions;

1) $(\alpha)_{i} \equiv(\alpha)_{i+1}$ for any $i<\operatorname{lh}(\alpha)$,

2) for any $i<\operatorname{lh}(\alpha)$ and for any $x$ such that $(\alpha)_{i} \leq x \leq(\alpha)_{i+1}$, either $x \equiv(\alpha)_{i}$ or $x \equiv(\alpha)_{i+1}$,

3) for any $x$ such that $(\alpha)_{l h(\alpha)} \leq x, x \equiv(\alpha)_{l h(\alpha)}$.

Let $C$ be the set of all essential chains. For $x \in M$, let $C(x)=\{\alpha ; \alpha \in C$ and $\left.(\alpha)_{1}=x\right\} . C(x)$ is nonempty for any $x \in M$. If $l h(\alpha)>1$, we write $\alpha^{+}$ for the chain $\left\langle(\alpha)_{2}, \ldots,(\alpha)_{l h(\alpha)}\right\rangle$. We define that 1) $k(x)=\max \{\operatorname{lh}(\alpha)$; $\alpha \in C(x)\}$ for $x \in M$ and 2) $k^{*}(\alpha)=k\left((\alpha)_{1}\right)$ for $\alpha \in C$. Obviously, $k(x) \geq 1$ for $x \in M$.

Lemma 3. 1) Let $\alpha \in C$. If $i<j \leq \operatorname{lh}(\alpha)$ then $(\alpha)_{i} \neq(\alpha)_{j}$.

2) There is an integer $n$ such that $l h(\alpha) \leq n$ for any $\alpha \in C$. 
3) $k^{*}\left(\alpha^{+}\right)<k^{*}(\alpha)$, and $k\left((\alpha)_{i}\right) \leq k^{*}(\alpha)$ if $i \leq \operatorname{lh}(\alpha)$.

Proof. 1) Let $(\alpha)_{i} \equiv(\alpha)_{j}$ for $i<j \leq l h(\alpha)$. Since $\alpha$ is a chain, $(\alpha)_{i}<(\alpha)_{i+1} \leq(\alpha)_{j}$. Then for any $B \in K$, i) if $W\left(B,(\alpha)_{i}\right)=t$ then $W\left(B,(\alpha)_{i+1}\right)=t$, and ii) if $W\left(B,(\alpha)_{i+1}\right)=t$ then $W\left(B,(\alpha)_{i}\right)=$ $W\left(B,(\alpha)_{j}\right)=t$. Thus $(\alpha)_{i} \equiv(\alpha)_{i+1}$. This contradicts Definition 2 .

2) Take the cardinality of $M / \equiv$ for $n$ and use 1 ).

3) Trivial.

Let $M_{0}$ be any Kripke model and $W_{0}$ be any $M_{0}$-valuation. Suppose $c \in M_{0}$ and $B$ be a formula in which only propositional variables $p_{1}, \ldots$, $p_{m}$ appear. Then the value of $W_{0}(B, c)$ depends only on the values of $W_{0}\left(p_{i}, b\right)$, where $1 \leq i \leq m$ and $c \leq b$. Keeping this fact in mind, we define two binary relations $\simeq($ on $M$ ) and $\sim($ on $C$ ). Intuitively, $x \simeq y$ means that the set $\left\{x^{\prime} ; x \leq x^{\prime}\right\}$ has the same structure as the set $\left\{y^{\prime}\right.$; $\left.y \leq y^{\prime}\right\}$ with respect to the value of $W$ for $K$. We first define $\simeq^{k}$ on $\{x ; k(x)=k$ and $x \in M\}$ and $\sim^{k}$ on $\left\{\alpha ; k^{*}(\alpha)=k\right.$ and $\left.\alpha \in C\right\}$ for any $k<\omega$, by the induction on $k$. 1) $x \simeq^{1} y$ if and only if $x \equiv y$ and $k(x)$ $=k(y)=1$. For $k>1, x \simeq^{k} y$ if and only if $x \equiv y, k(x)=k(y)=k, x R y$ and $y R x$, where $u R v$ means that for any $\alpha \in C(u)$ there is an $h$ and $\beta \in C(v)$ such that $\alpha^{+} \sim^{h} \beta^{+}$. 2) $\alpha \sim^{k} \beta$ if and only if $\operatorname{lh}(\alpha)=\operatorname{lh}(\beta), k^{*}(\alpha)$ $=k^{*}(\beta)=k$ and for any $i \leq l h(\alpha)$, there is a $k_{i}$ such that $(\alpha)_{i} \simeq^{k_{2}}(\beta)_{i}$.

Now, we define $\simeq$ and $\sim$ as follows.

$x \simeq y$ if and only if there is a $k$ such that $x \simeq^{k} y$, and

$\alpha \sim \beta$ if and only if there is a $k$ such that $\alpha \sim^{k} \beta$.

We show that $\simeq$ and $\sim$ are well-defined. $W D(k)$ (or $W D^{*}(k)$ ) means that for any $h \leq k$ the relation $\simeq^{h}$ (or $\sim^{h}$ ) is well-defined. Then it is easy to see that 1) $W D(1)$ holds, 2) $W D(k)$ implies $W D^{*}(k)$ and 3) $W D^{*}(k)$ implies $W D(k+1)$, by using Lemma 3,3$)$. So, both $W D(k)$ and $W D^{*}(k)$ hold for any $k$. Hereafter, we sometimes use the induction of this kind.

Lemma 4. $\simeq($ or $\sim)$ is an equivalence relation over $M($ or $C$, respectively). 
Lemma 5. $M / \simeq$ is finite.

Proof. We write $[x]$ (or $\langle\alpha\rangle$ ) for the element of $M / \simeq$ (or $C / \sim$ ) which contains $x$ (or $\alpha$, respectively). Let $D_{n}=\{[x] ; k(x)=n\}$ and $E_{n}=\left\{\langle\alpha\rangle ; k^{*}(\alpha)=n\right\} . \quad F(k)$ (or $F^{*}(k)$ ) means that $D_{n}$ (or $E_{n}$, respectively) is finite for any $n \leq k$. Then

1) $F(1)$ holds, since $\overline{\bar{D}}_{1} \leq \overline{\overline{M /}} \equiv<\omega$,

2) $F(k)$ implies $F^{*}(k)$, since $\overline{\bar{E}}_{i} \leq \sum_{j \leq i}\left(\overline{\bar{D}}_{1} \times \cdots \times \bar{D}_{j}\right)<\omega$ for $i \leq k$,

3) $F^{*}(k)$ implies $F(k+1)$, since $\overline{\bar{D}}_{i+1}^{j \leq i} \leq \overline{\overline{M /}} \times \overline{\left.\overline{P\left(\bigcup_{j \leq i} E_{j}\right.}\right)}<\omega$ for $i \leq k$, where $\overline{\bar{G}}$ denotes the cardinality of a set $G$ and $P(G) \stackrel{j \leq i}{\text { denotes the power }}$ set of $G$. Thus, $F(k)$ holds for any $k$ and hence $M / \simeq$ is finite, since $M / \simeq=\bigcup_{n \leq k} D_{n}$ for some $k$ by Lemma 3,2$)$.

Next, we define a binary relation $\leq^{*}$ on $M / \simeq$ as follows. $[x] \leq^{*}[y]$ if and only if there is an $\alpha \in C(x)$ such that $(\alpha)_{i} \simeq y$ for some $i \leq \operatorname{lh}(\alpha)$.

Lemma 6. The relation $\leq^{*}$ is well-defined and is an order relation on $M / \simeq$.

Proof. We first show that if $x^{\prime} \simeq x, y^{\prime} \simeq y$ and $[x] \leq^{*}[y]$, then $\left[x^{\prime}\right] \leq^{*}\left[y^{\prime}\right]$. Let $\alpha \in C(x)$ and $(\alpha)_{i} \simeq y$ for some $i \leq l h(\alpha)$. If $\operatorname{lh}(\alpha)=1$ or $i=1$, then $x=(\alpha)_{1} \simeq y$. By the assumption, $x^{\prime} \simeq y^{\prime}$. Let $\beta$ be any element in $C\left(x^{\prime}\right)$. Then $(\beta)_{1} \simeq y^{\prime}$. Thus $\left[x^{\prime}\right] \leq^{*}\left[y^{\prime}\right]$. Suppose that $l h(\alpha)>1$ and $i>1$. Since $x^{\prime} \simeq x$ and $k(x) \geq l h(\alpha)>1$, there is a $\beta \in C\left(x^{\prime}\right)$ such that $\alpha^{+} \sim \beta^{+}$. Let $z=\left(\beta^{+}\right)_{i-1}$. Then $z \simeq\left(\alpha^{+}\right)_{i-1}=(\alpha)_{i} \simeq y \simeq y^{\prime}$. Since $(\beta)_{i}=z,\left[x^{\prime}\right] \leq^{*}\left[y^{\prime}\right]$. Next, we show $\leq^{*}$ is an order relation on $M / \simeq$. It is trivial that $\leq^{*}$ is reflexive. Suppose that $[x] \leq^{*}[y]$ and $[y] \leq^{*}[x]$. Let $\alpha \in C(x)$ and $(\alpha)_{i} \simeq y$. Since $\left[(\alpha)_{i}\right] \leq *[x]$, there is a $\beta \in C$ such that $(\beta)_{1}=(\alpha)_{i}$ and $(\beta)_{j} \simeq x$ for some $j$. Define $\gamma \in C$ as follows:

$$
(\gamma)_{k}= \begin{cases}(\alpha)_{k} & \text { if } k \leq i \\ (\beta)_{k-i+1} & \text { if } i<k \leq \operatorname{lh}(\beta)+i-1 .\end{cases}
$$


Then $(\gamma)_{1}=x \simeq(\beta)_{j}=(\gamma)_{i, j-1}$. So $(\gamma)_{1} \equiv(\gamma)_{i_{r}-1}$. By Lemma 3,1$), i+j$ $=2$. Thus $i=1$ and hence $x=(\alpha)_{1} \simeq y$. This means $\leq^{*}$ is asymmetric. The transitivity of $\leq^{*}$ is proved similarly.

Now, define a Kripke model $N$ by the set $M / \simeq$ with the relation $\leq^{*}$.

Lemma 7. $L(M)<L(N)$.

Proof. Define a mapping $f$ from $M$ to $N$ by $f(x)=[x]$. We show that $f$ is an embedding of $M$ into $N$ (see [4]). Let $x \leq y$. Then there is an $\alpha \in C(x)$ such that $(\alpha)_{i} \simeq y$ for some $i$. Thus $f(x) \leq^{*} f(y)$. Next, suppose $f(x) \leq *[y]$. By the definition, there is an $\alpha \in C(x)$ such that $(\alpha)_{i} \simeq y$ for some $i$. So, $x \leq(\alpha)_{i}$ and $f\left((\alpha)_{i}\right)=[y]$. Now our lemma follows from Theorem $2.11[4]$.

Lemma 8. $A \notin L(N)$.

Proof. Define an $N$-valuation $V$ by $V(p,[x])=W(p, x)$ for any propositional variable $p$ in $K$. We remark that the proof in the following can be carried out irrespective of the value of $V(q,[x])$ for any propositional variable $q$ not in $K . \quad V$ is well-defined, since $x \simeq y$ implies $x \equiv y$. It can be easily proved that $V$ is really an $N$-valuation. We show that $V(B,[x])=\mathbb{W}(B, x)$ for any $B \in K$, by the induction on the number of the logical connectives in $B$. We show this, when $B$ is of the form $C>D$. Let $W(B, x)=t$. Suppose that $[x] \leq^{*}[y]$ and $V(C,[y])=t$. Then by the proof of Lemma 3 it follows that there is a $y^{\prime}$ such that $x \leq y^{\prime}$ and $y^{\prime} \simeq y$. So, $W\left(B, y^{\prime}\right)=t$. By the hypothesis of the induction, $W\left(C, y^{\prime}\right)=t$ and hence $W\left(D, y^{\prime}\right)=t$. Thus $V(D,[y])=V\left(D,\left[y^{\prime}\right]\right)=t$. So, $V(B,[x])=t$. Next, suppose $W(B, x)=f$. Then there is a $y$ such that $x \leq y, W(C, y)=t$ and $W(D, y)=f$. So, $[x] \leq *[y], V(C,[y])=t$ and $V(D,[y])=f$ by the hypothesis. Thus $V(B,[x])=f$. In other cases, the proof is easy. Now, taking $A$ for $B$ and $a$ for $x$, we get 
$V(A,[a])=W(A, a)=f$. Thus $A \notin L(N)$.

Using Lemma 5,7 and 8 it is proved that for each $A \notin L(M)$ there is a finite Kripke model $N_{A}$ such that $A \notin L\left(N_{A}\right)$ and $L(M) \subset L\left(N_{A}\right)$. So, $L(M) \bigcap_{A \notin L(M)} L\left(N_{A}\right)$. Now, the proof of Theorem 1 is completed, by using Corollary $1.3[4]$.

Corollary 9. Let $L$ be any intermediate logic. Then the following two conditions are equivalent.

1) L has a (characteristic) Kripke model.

2) $L$ has the finite model property.

Proof. 1) implies 2) by Theorem 1. We show that 2) implies 1). Since for any finite pseudo-Boolean model $P$ there is a finite Kripke model $M$ such that $P)(L(M)$ by Corollary $1.5[4], L) C \bigcap_{i \in l} L\left(M_{i}\right)$ for some finite Kripke models $M_{i}$. By Corollary $2.8[4], L\left(\left(M_{i}\right)_{i \in l}\right)>C \bigcap_{i \in I}$ $L\left(M_{i}\right)$. Hence $L$ has a Kripke model $\left(M_{i}\right)_{i \in I}$.

For $n<\omega$, we write $P_{n}$ for the pseudo-Boolean algebra $P_{\left(R_{n \omega}\right)}$ (see [4]), which is a model of $L P_{n}$.

Corollary 10. Let $L$ be any intermediate logic in $\mathscr{S}_{n}(n<\omega)$. Then following condition 3) is equivalent to 1) (or 2)) in Corollary 9.

3) There are finite subalgebras $Q_{i}(i \in I)$ of $P_{n}$ such that $L X \bigcap_{i \in I} Q_{i}$.

Proof. It is obvious that 3 ) implies 2). We show 1) implies 3 ). By Theorem 1 and Theorem 2.10 [4], if $L$ has a Kripke model, then there are finite Kripke models $M_{i}$ 's having the least element (as partially ordered sets) such that $L \supset \subset \bigcap_{i \in I} L\left(M_{i}\right)$. By the discussion in $\S 4[4]$, we can show that $R_{n_{\omega}}$ is embeddable into any finite Kripke model $N$ such that $h(N) \leq n$ and $N$ has the least element. Let $Q_{i}=P_{M_{i}}$. Then $Q_{i}$ is a subalgebra of $P_{n}$ by Theorem 4.6 in de Jongh-Troelstra [2]. So 3) holds, since $\left.Q_{i}\right)\left(L\left(M_{i}\right)\right.$.

Now we extend Corollary 9 to that for logics between Johansson's 
minimal logic $L M$ and the classical logic $L K$. We write $\mathscr{L}$ for the set of all logics between $L M$ and $L K$. A pair $(P, a)$ is said to be a lattice model (for $\mathscr{L}$ ), if $P$ is a relatively pseudo-complemented lattice and $a \in P$ ( $a$ gives the interpretation of $\Lambda$, where $\Lambda$ is a proposition used for the definition of the negation of a formula). If $P$ has the zero element (i.e, the least element) 0 , then $P$ is a pseudo-Boolean algebra. So, in such a case, a lattice model $(P, 0)$ is equivalent to a pseudo-Boolean model $P$. We remark that any finite relatively pseudo-complemented lattice is a pseudo-Boolean algebra. We write $L^{*}(P, a)$ for the set of all formulas valid in $(P, a)$. It is well-known that any logic $L$ in $\mathscr{L}$ has a characteristic lattice model, i.e., $L \backslash L^{*}(P, a)$ for some lattice model $(P, a)$. We now define Kripke-type models for $\mathscr{L}$, following Segerberg [5].

Definition 11. A pair $(M, Q)$ is a generalized Kripke model (abbreviated as GK-model) if and only if $M$ is a partially ordered set and $Q$ is a closed subset of $M$, i.e., for any $u, v \in M,(u \in Q$ and $u \leq v)$ implies $v \in Q$. An $(M, Q)$-valuation $W$ is defined similarly as an M-valuation except in the case that a formula is of the form $\neg A$. That is, instead of 5) in Definition 1.1 in [4], we use $5^{\prime}$ ).

$\left.5^{\prime}\right) W(\neg A, u)=t$ if and only if for any $r$ in $M$ such that $u \leq r$, $W(A, r)=f$ or $r \in Q$.

If $Q$ is empty, then a GK-model $(M, Q)$ is nothing else but a Kripke model $M$. Let $L(M, Q)$ be the set of all formulas valid in $(M, Q)$. It is easy to verify that $L(M, Q) \in \mathscr{L}$. Following theorem can be proved similarly as Corollary 1.3 and 1.5 in $[4]$.

Theorem 12. 1) For any $G K$-model $(M, Q), L(M, Q) \backslash L^{*}\left(P_{M}, Q\right)$.

2) Let $(P, a)$ be any lattice model such that $P$ is finite. Then $L^{*}(P, a) \supset C\left(M_{P},\left\{F ; a \in F \in M_{P}\right\}\right)$.

Let $(M, Q)$ and $\left(N, Q^{\prime}\right)$ be GK-models. A mapping $f$ from $M$ to $N$ is said to be an embedding of $(M, Q)$ into $\left(N, Q^{\prime}\right)$ if $f$ is an embedding 
of $M$ into $N$ (see [4]) and $f^{-1}\left(Q^{\prime}\right)=Q$. Now, we obtain a result analogous to Theorem 2.11 [4].

$\left.{ }^{*}\right)$ If there is an embedding of $(M, Q)$ into $\left(N, Q^{\prime}\right)$ then $L(M, Q) C$ $L\left(N, Q^{\prime}\right)$.

Now, using $(*)$, we can extend Theorem 1 and Corollary 9. A logic $L$ in $\mathscr{L}$ is said to have the finite model property, if there are lattice models $\left(P_{i}, a_{i}\right)(i \in I)$ such that each $P_{i}$ is finite and $\left.L\right) \subset \bigcap_{i \in l} L^{*}\left(P_{i}, a_{i}\right)$.

Theorem 13. Let $L$ be any logic in $\mathscr{L}$. If $L$ has a characteristic $G K$-model then $L$ has the finite model property.

Proof. Let $x \equiv^{\prime} y$, if $x \equiv y$ and $(x \in Q$ if and only if $y \in Q$ ). Replace $\equiv$ by $\equiv^{\prime}$ in any occurrence of $\equiv$ in the proof of Theorem 1 . After these replacements, all lemmas from 2 to 6 hold also. Instead of Lemma 7 and 8, we can show that $L(M, Q) \subset L\left(N, Q^{\prime}\right)$ and $A \notin L\left(N, Q^{\prime}\right)$, by defining $Q^{\prime}=\{[x] ; x \in Q\}$ and using $\left(^{*}\right)$.

Corollary 14. Let $L \in \mathscr{L}$. Then the following two conditions are equivalent.

1) L has a GK-model.

2) $L$ has the finite model property.

Proof. By Theorem 13, 1) implies 2). Suppose that 2) holds. By Theorem 12, there are GK-models $\left(M_{i}, Q_{i}\right)$ such that $L \backslash C \bigcap_{i \in I} L\left(M_{i}, Q_{i}\right)$. We can show $\bigcap_{i \in I} L\left(M_{i}, Q_{i}\right) \supset C L\left(\left(M_{i}\right)_{i \in I}, \bigcup_{i \in I} Q_{i}\right)$. Thus 1) holds.

By Theorem 13 and Harrop's theorem [4], we can prove that if a logic $L$ in $\mathscr{L}$ is finitely axiomatizable and has a GK-model then $L$ is decidable. In particular, all logics studied in [5] satisfy the above premises. Thus they are decidable.

Note Added in Proof (June 20, 1971): Recently, the author has known the result by Jankov [6]. He showed that there exists an intermediate logic not having the finite model property. 


\section{References}

[1] Harrop, R., On the existence of finite models and decision procedures for propositional calculi, Proc. Cambridge Phil. Soc. 54 (1958) 1-13.

[2] De Jongh, D.H.J. and A.S. Troelstra, On the connection of partially ordered sets with some pseudo-Boolean algebras, Indag. Math. 28 (1966) 317-329.

[3] Lemmon, E. J., Algebraic semantics for modal logics II, J. Symbolic Logic, 31 (1966) 191-218.

[4] Ono, H,. Kripke models and intermediate logics, Publ. RIMS, Kyoto Univ. 6 (1970/71), 461-476.

[5] Segerberg, K., Propositional logics related to Heyting's and Johansson's, Theoria, 34 (1968) $26-61$.

[6] Jankov, V.A., Constructing a sequence of strongly independent superintuitionistic propositional calculi, Soviet Math. Dokl. 9 (1968) 806-807.

\section{Correction (Added July 21, 1971)}

By a personal communication from C. Smorynski, we knew an error in the above Lemma 7, which caused the main theorem impossible. But a minute examination of the proof brought us the conclusion that the theorem holds if we restrict our arguments to the finite slices. The corrected proof runs similarly by changing the definitions of $\simeq$ and $\leq^{*}$ as follows.

1) $x \simeq y$ if $x \equiv y$ and $d(0, x)=d(0, y)$, where 0 is the least element of $M$ whose existence does not restrict the generality by Theorem 2.10 of $[4]$, and either i) both $x$ and $y$ are maximal or ii) neither $x$ nor $y$ are maximal and $x R y$ and $y R x$, where $x R y$ means $\forall u(x<u)$ $\exists v(u \simeq v$ and $y<v)$ ).

2) $[x] \leq^{*}[y]$ if for any $x^{\prime} \simeq x$ there is $y^{\prime}$ such that $y^{\prime} \simeq y$ and $x^{\prime} \leq y^{\prime}$. 
